\title{
High-Performance Two-Wavelength Asymmetric Fabry-Perot Modulator with a Decoupled Cavity Design
}

\author{
Chia-Ming Tsai and Chien-Ping Lee, Senior Member, IEEE
}

\begin{abstract}
We have demonstrated a high-performance twowavelength asymmetric Fabry-Perot modulator (AFPM) by using a novel decoupled cavity design. In this new design, the middle mirror supplies a back-mirror reflectivity for the shortwavelength modulator and, at the same time, a front-mirror reflectivity for the long-wavelength modulator. The front mirror is designed to be transparent at the long wavelength and reflective at the short wavelength to eliminate the interference between the two modulators. With this decoupled cavity design, we greatly simplified the matching requirement for the two cavities and obtain much more flexibility in the device design. Experimentally, we have demonstrated a two-wavelength modulator operated at 860 and $890 \mathrm{~nm}$ with operating voltages of 3.4 and $6.2 \mathrm{~V}$ and reflectivity changes of $62 \%$ and $44 \%$, respectively.
\end{abstract}

Index Terms - Fabry-Perot resonators, optical switches, quantum-confined Stark effect, quantum-well devices, wavelength-division multiplexing.

\section{INTRODUCTION}

$\mathbf{O}$ PTICAL switching is essential in optical communications, optical interconnects, and optical signal-processing systems. Reflection-type multiple-quantum-well (MQW) optical modulators have been extensively studied for such applications [1]-[6]. The surface-normal geometry of such devices also provides the possibility of two-dimensional (2D) array configuration for parallel optical signal processing. For applications mentioned above, a high data throughput is very important. The use of multiple wavelengths, therefore, becomes attractive. Most of the modulators reported, however, were designed for single-wavelength operation. We have recently reported for the first time two-wavelength quantumwell modulators [7], [8]. These devices were based on two coupled cavities with two absorption regions. Two different design schemes including the simple cavity design [7] and the balanced cavity design [8] have been demonstrated and both of them have shown good modulation characteristics. In this paper, we report an improved structure which utilizes two decoupled cavities. Excellent modulation performance has been achieved.

Manuscript received April 21, 1997. This work was supported by the National Science Council of the Republic of China under Contract NSC862215-E009-010.

The authors are with the Department of Electronics Engineering and Institute of Electronics, National Chiao Tung University, Hsin-Chu 300, Taiwan, Republic of China.

Publisher Item Identifier S 0018-9197(98)01775-8.
A two-wavelength modulator based on two absorption regions can be schematically shown in Fig. 1(a). The absorption region for the short wavelength $\lambda_{1}$ is put above (or before) the absorption region for the long wavelength $\lambda_{2}$. In this way, the short-wavelength light will not be blocked by the long-wavelength absorption region. Three mirrors are put in the front, in the back, and in the middle of the absorption regions to form two Fabry-Perot cavities. Because the longwavelength absorption region has a strong absorption for the short-wavelength light, the $\lambda_{1}$ absorbing region can be treated as if it is confined in between the front mirror $R_{f}$ and the middle mirror $R_{m}$. But, for operation at $\lambda_{2}$, the $\lambda_{2}$ absorbing layer is confined between the the back mirror $R_{b}$ and the effective front mirror $R_{f}^{\lambda 2}$, which includes the middle mirror and the front cavity. In the previous two designs we have studied, the front cavity plays a very important role for achieving the whole two-wavelength operation. It determines the operations of both the short wavelength and the long wavelength. As a result, both of these two designs unavoidably suffer from complicated matching requirements in the front cavity. In this paper, we present a novel decoupled cavity design which can efficiently reduce the interference between the two wavelengths.

\section{DeCoupled CAVITY Design}

As we can see from Fig. 1(a), the front cavity serves as the front mirror for the long wavelength, $\lambda_{2}$. If the front cavity is not properly designed or a slight variation in layer parameters occurs during device growth, the overall performance for operation at $\lambda_{2}$ is degraded. To solve this problem, we use, instead of a conventional broad-band mirror, a filter-type front mirror which is transparent at $\lambda_{2}$ but reflective at $\lambda_{1}$. With this simple modification, the disturbance on $\lambda_{2}$ operation caused by the light reflection from the front mirror can be totally eliminated. The front cavity simply becomes a transparent region for $\lambda_{2}$. The corresponding reflection characteristic for each mirror of this decoupled cavity design is shown in Fig. 1(b). In this design, the length of the front cavity needs only to satisfy the $\lambda_{1}$ resonant condition and the $\lambda_{2}$ resonant condition is only determined by the rear cavity. It greatly reduces the difficulty in device growth, especially for devices that need more precise wavelength control, such as normally off modulators and SEED's. It also provides more flexibility 


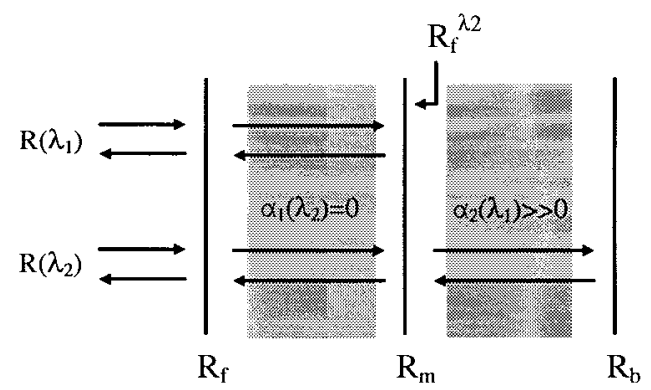

(a)

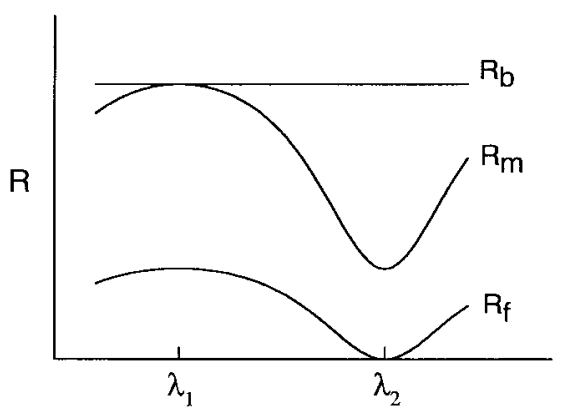

(b)

Fig. 1. (a) Schematic of two-wavelength modulator and (b) the mirror reflective properties for the decoupled cavity design.

in the cavity design for each wavelength since the coupling effect between the two cavities is eliminated.

\section{EXPERIMENTAL RESULTS}

In this paper, the short-wavelength absorbing region is GaAs-AlGaAs QW's designed for operation at $860 \mathrm{~nm}\left(\lambda_{1}\right)$, and the long-wavelength absorbing region is InGaAs-AlGaAs QW's designed for operation at $890 \mathrm{~nm}\left(\lambda_{2}\right)$.

In the present design, the most important thing is to have the right reflection characteristics for the middle mirror $R_{m}$ and the front mirror $R_{f}$. Considering the matching requirement for the rear $\lambda_{2}$ cavity, we chose to use $\lambda_{2} / 4$-based distributed Bragg reflector (DBR) stacks to construct the desired middle mirror. It consisted of two face-to-face DBR stacks, one with six pairs of $\mathrm{AlAs}-\mathrm{Al}_{0.1} \mathrm{Ga}_{0.9} \mathrm{As}$ layers and the other one 12 pairs. The calculated reflection spectrum is plotted in Fig. 2. The calculated reflectivity is $57 \%$ for $890 \mathrm{~nm}$ and $95 \%$ for 860 $\mathrm{nm}$. For the front mirror, we used $\lambda_{1} / 4$-based DBR stacks. A three-pair $\mathrm{AlAs}-\mathrm{Al}_{0.1} \mathrm{Ga}_{0.9} \mathrm{As} \lambda_{1} / 4$ stack with an extra $5 \lambda_{1^{-}}$ thick $\mathrm{Al}_{0.1} \mathrm{Ga}_{0.9} \mathrm{As}$ extended layer on top was used. The extra $5 \lambda_{1}$-thick $\mathrm{Al}_{0.1} \mathrm{Ga}_{0.9}$ As layer was for the purpose of creating a transparent window at $890 \mathrm{~nm}$ while maintaining a high reflectivity at $860 \mathrm{~nm}$. The calculated reflection spectrum is also plotted in Fig. 2. As we can see from the figure, it has a reflectivity of $66 \%$ at $\lambda_{1}$ and is almost transparent at $\lambda_{2}$. This satisfies the requirement for a decoupled cavity design.

Based on the mirror design described above, we can calculate the critical absorption strength (the absorption coefficient times the thickness of the absorption region) to achieve zero reflection for each wavelength. For $\lambda_{1}$, it is 0.182 . For $\lambda_{2}$, if we use the same back mirror reflectivity, i.e., $R_{b}=$ 0.95 , the required absorption strength is 0.255 . So based on

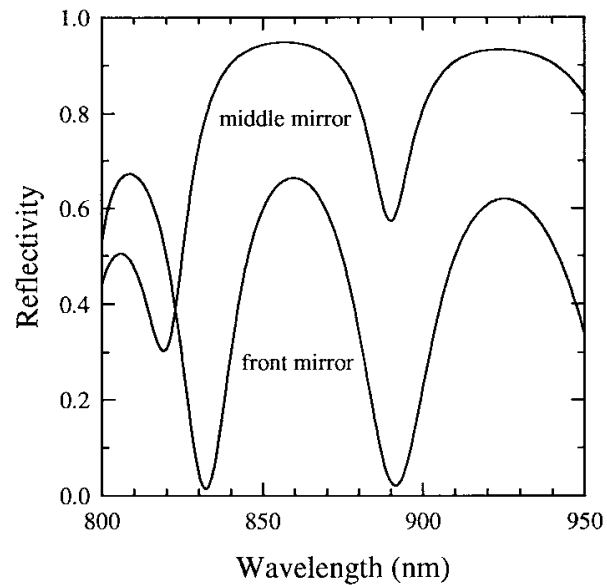

Fig. 2. Calculated reflection spectra of the middle mirror and the front mirror.

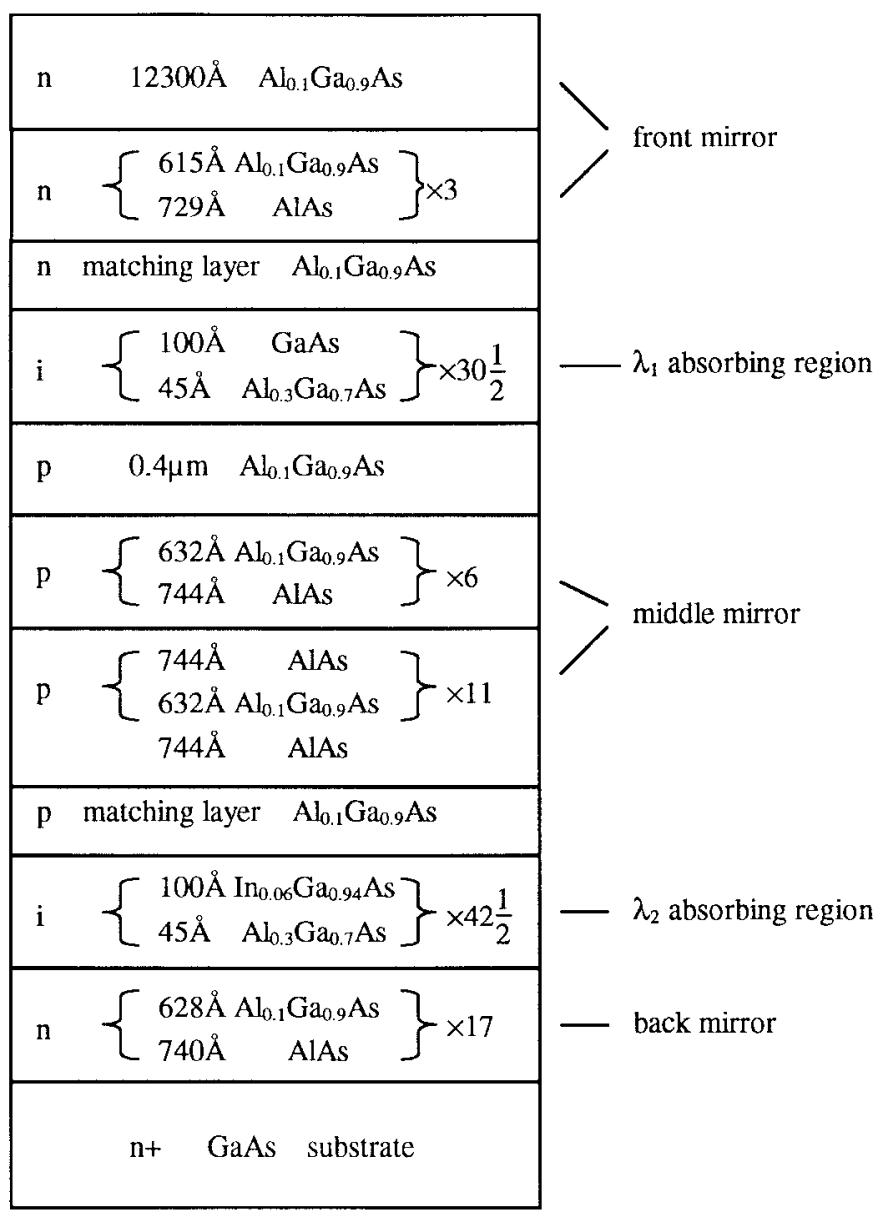

Fig. 3. Grown device structure of the two-wavelength modulator with the decoupled cavity design.

these numbers, we can easily design each absorption region accordingly to complete the whole device design.

After the above design procedure, we fabricated a twowavelength modulator with the device structure shown in Fig. 3. All layers were grown by a MBE system with in situ reflection monitoring to ensure correct layer growth. Measured reflection spectra after growth of each critical region are shown 


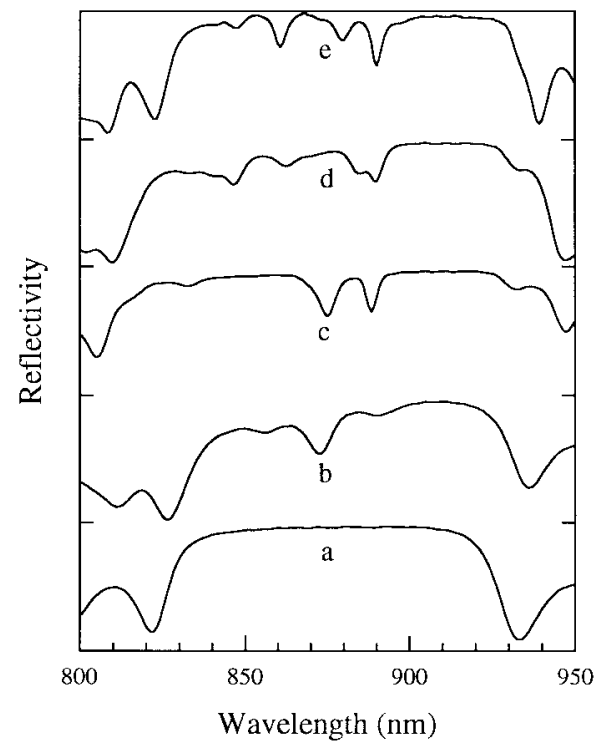

Fig. 4. In situ monitored reflection spectra after growth of (a) the back mirror, (b) the p- $\mathrm{Al}_{0.1} \mathrm{Ga}_{0.9} \mathrm{As}$ matching layer, (c) the middle mirror, (d) the $\mathrm{n}-\mathrm{Al}_{0.1} \mathrm{Ga}_{0.9}$ As matching layer, and (e) the front mirror.

in Fig. 4. The growth began with a back mirror consisting of a 17-pair n-AlAs(628 $\AA)-\mathrm{Al}_{0.1} \mathrm{Ga}_{0.9} \mathrm{As}(740 \AA)$ DBR stack. The calculated reflectivity at $890 \mathrm{~nm}$ is over $95 \%$. Curve (a) in Fig. 4 shows the in situ measured reflection spectrum. Then a 40-period undoped $\operatorname{In}_{0.06} \mathrm{Ga}_{0.94} \mathrm{As}(100 \AA)-\mathrm{Al}_{0.3} \mathrm{Ga}_{0.7} \mathrm{As}(45$ $\AA$ ) QW absorbing region was grown on top of the back mirror. During this growth, the substrate temperature was kept at $500{ }^{\circ} \mathrm{C}$ to avoid the formation of dislocations caused by strain relaxation. Before the growth of the middle mirror, a $\mathrm{p}-\mathrm{Al}_{0.1} \mathrm{Ga}_{0.9} \mathrm{As}$ matching layer was grown to ensure the longwavelength operation at $890 \mathrm{~nm}$. The reflectivity of the whole structure was monitored during the growth of this layer until we began to see a clear resonant dip at $890 \mathrm{~nm}$, which is shown by curve (b) in Fig. 3. From this spectrum, we also identified the position of the nonbiased exciton at a wavelength of 873 $\mathrm{nm}$. A middle mirror with the cavity structure mentioned before was then grown on top of this resonance-matched diode structure and the in situ measured overall reflection spectrum is shown by curve (c). Then we grew a 30-period undoped $\operatorname{GaAs}(100 \AA)-\mathrm{Al}_{0.3} \mathrm{Ga}_{0.7} \mathrm{As}(45 \AA) \mathrm{QW}$ absorbing region for $\lambda_{1}$. Another matching layer of $\mathrm{n}-\mathrm{Al}_{0.1} \mathrm{Ga}_{0.9} \mathrm{As}$ was grown for resonance at $860 \mathrm{~nm}$. The resonance can be clearly seen in the reflection spectrum shown by curve (d). From this curve, we also identified the position of the exciton of the shortwavelength quantum well to be at $848 \mathrm{~nm}$. Growth of a front mirror with the structure mentioned above finally completed the whole device structure. The overall reflection spectrum was shown as curve (e). From this spectrum, clear resonant behaviors were observed at both wavelengths of 860 and 890 $\mathrm{nm}$. The completed structure has an n-i-p-i-n configuration.

After the growth, we fabricated the device by two-step etching to separately define the three ohmic contacts for both the top and the substrate n-layers as well as the middle player. The top optical window was $(200 \mu \mathrm{m})^{2}$. Fig. 5 shows the measured modulation spectra for both wavelengths. For the short-wavelength operation at $860 \mathrm{~nm}$, an operating voltage as

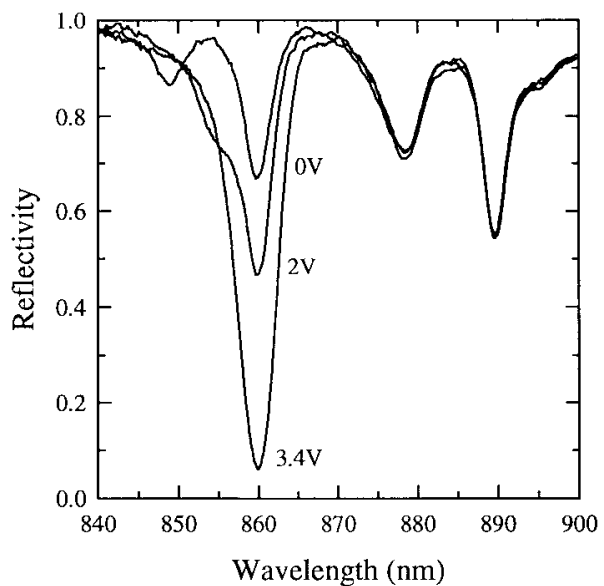

(a)

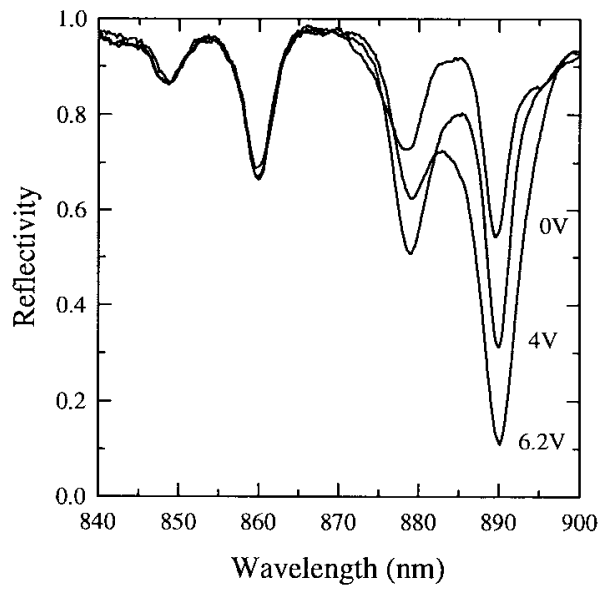

(b)

Fig. 5. Measured modulation spectra of the fabricated two-wavelength modulator with separate bias applied on (a) the upper short-wavelength diode and (b) the lower long-wavelength diode.

low as $3.4 \mathrm{~V}$ and a reflectivity change of $62 \%$ were obtained. For the long-wavelength operation, an operating voltage of $6.2 \mathrm{~V}$ and a reflectivity change of $44 \%$ were obtained at 890 $\mathrm{nm}$. The relatively large operating voltage for $890 \mathrm{~nm}$ resulted from a thicker long-wavelength QW absorbing layer and a larger wavelength separation between the excitonic and the resonant position.

\section{CONCLUSION}

We have successfully developed a new decoupled cavity design for implementation of two-wavelength modulators by using filter-type mirrors as the middle and the front mirrors. With this design, one can completely isolate the operating cavity for each wavelength from each other and achieve much more flexibility in the whole device design. The matching procedure for the two-wavelength operation becomes two independent steps: the front cavity satisfies the $\lambda_{1}$ resonance and the rear cavity satisfies the $\lambda_{2}$ resonance. Therefore, the whole structure is like two independent modulators grown sequentially together without any interference between each other. 
Experimentally, a short-wavelength cavity with the frontand the back-mirror reflectivities of $66 \%$ and $95 \%$ was designed for $860 \mathrm{~nm}$. An operating voltage of $3.4 \mathrm{~V}$ and a reflectivity change of $62 \%$ were obtained. For the longwavelength operation at $890 \mathrm{~nm}$, a cavity with an effective front- and back-mirror reflectivities of $57 \%$ and $95 \%$ was adapted. An operating voltage of $6.2 \mathrm{~V}$ as well as a reflectivity change of $44 \%$ were obtained.

\section{REFERENCES}

[1] C. C. Barron, C. J. Mahon, B. J. Thibeault, G. Wang, W. Jiang, L. A. Coldren, and J. E. Bowers, "Millimeter-wave asymmetric Fabry-Perot modulators," IEEE J. Quantum Electron., vol. 31, pp. 1484-1493, 1995.

[2] M. R. Feldman, S. C. Esener, C. C. Guest, and S. H. Lee, "Comparison between optical and electrical interconnects based on power and speed considerations," Appl. Opt., vol. 27, no. 9, pp. 1742-1751, 1988.

[3] C. Fan, B. Mansoorian, D. A. Van Blerkom, M. W. Hansen, V. H Ozguz, S. C. Esener, and G. C. Marsden, "Digital free-space optical interconnections: A comparison of transmitter technologies," Appl. Opt., vol. 34, no. 17, pp. 3103-3115, 1995.
[4] See IEEE J. Select. Topics Quantum Electron., vol. 2, 1996.

[5] A. L. Lentine and D. A. B. Miller, "Evolution of the SEED technology: Bistable logic gates to optoelectronic smart pixels," IEEE J. Quantum Electron., vol. 29, pp. 655-669, 1993.

[6] D. A. B. Miller, "Novel analog self-electrooptic-effect devices," IEEE J. Quantum. Electron., vol. 29, pp. 678-698, 1993.

[7] C. M. Tsai and C. P. Lee, "Novel reflection-type normally-on twowavelength modulator," Electron. Lett., vol. 33, no. 7, pp. 611-613, 1997.

[8] _ "Normally-on reflection-type two-wavelength quantum well modulator with balanced cavity design," IEEE Photon. Technol. Lett., vol. 9, pp. 928-930, 1997.

Chia-Ming Tsai, photograph and biography not available at the time of publication.

Chien-Ping Lee (M'80-SM'94), photograph and biography not available at the time of publication. 